8. Audiometry showed left conductive hearing loss. CT showed the fusion of the head of malleus and the wall of epitympanum in left side. He underwent left type I tympanoplasty, and got an effective hearing level. However, after 6 months, the hearing level in the left ear was the same as preoperative one. The recurrence of fixation of the malleus head was suspected.

Case2: The case was 9-year-old girl. She had recurrent otitis media at the age of 3 . Though her otitis media was improved, she had still left conductive hearing loss. Thus, she had been referred to our department at the age of 6. CT showed the fusion of the head of malleus and the wall of epitympanum in left side. She underwent left type IIIc tympanoplasty, and got an effective hearing level.

Conclusion: It was suggested that type IIIc tympanoplasty is more appropriate approach for a malleus ankylosis than type I tympanoplasty.

doi:10.1017/S0022215116006848

\section{ID: IP188}

The improvement of the bone hearing thresholds after removing cholesteatoma from the round window: our experience Salii O. V. 1, Verchovtseva L. I. 1, Tarasevich T. N. 2

Presenting Author: Olga Salii

Olga Salii ${ }^{1}$, Tatyana Tarasevich ${ }^{2}$

${ }^{1}$ Regional Clinical Hospital №1, Yekaterinburg, ${ }^{2}$ Institute of Immunology and Physiology, Ural Branch of the Russian

Academy of Sciences, Yekaterinburg

\section{Learning Objectives:}

Background: $30 \%$ of all patients treated in our ENT-department are patients with the pathology of middle ear. Out of all our patients with chronic otitis media $54 \%$ have cholesteatoma. Hearing function recovering is considered as impotent as complete cholesteatoma removing.

In some cases of cholesteatoma it was observed that after surgery there is an improvement not only of sound conduction but also of sound perception.

Objective: The investigation of possible reasons of sound perception improvement of patients after removing cholesteatoma with tympanoplasty.

Materials and methods: An assessment of 256 patients hearing results was carried out retrospectively. The patients underwent surgery on account of chronic otitis media with cholesteatoma from 2009 to 2015 . Hearing assessment was analyzed by data mean value for 4 frequencies: 500 ; Hz, $1000 ; \mathrm{Hz}, 2000 ; \mathrm{Hz}, 4000 ; \mathrm{Hz}$ before surgery and 3 and 6 months after surgery. Moreover, air-bone interval, air-conductive thresholds and bone-conductive thresholds were assessed before and after surgery, and the absolute increase of air conduction was measured after surgery.
Carefully recoded surgery protocols were analyzed.

Results and discussion: According to the analysis of data it was found that an improvement of hearing thresholds mean value occurs not only for air conduction but also for bone conduction in $32 \%$ of the cases. All patients were divided in 2 groups: with increasing bone conduction and without increasing bone conduction. Several points in these groups were analyzed.

According to the analysis of surgeries' protocols, in $87 \%$ of cases there was sound perception improvement of those patients who had cholesteatoma localized in the round window area and had it completely removed during surgery.

Conclusions: Removing cholesteatoma from the round window region promotes sound perception improvement due to the free movement of the round window membrane that, in its turn, improves the movement of perilymph.

\section{doi:10.1017/S002221511600685X}

\section{ID: IP189}

Sigmoid sinus thrombosis and facial paralysis associated to mastoiditis: A case report

\section{Presenting Author: Oana Alexandra Sandu}

Oana Alexandra Sandu ${ }^{1}$, Codrut Saraforanu ${ }^{2}$

1 "Carol Davila" University of Medicine and Pharmacy, 21 "Carol Davila" University of Medicine and Pharmacy, Bucharest, Romania, ${ }^{2}$ ENT\&HNS Department, "Sfanta Maria" Hospital, Bucharest, Romania

Learning Objectives: Initial surgical approach (antromastoidectomy) was not appropriate for this case. Right mastoidectomy, broad-spectrum antibiotics and anticoagulants has been the treatment of choice.

Introduction: Otogenic sigmoid sinus thrombosis is a rare complication of mastoiditis. This paper aims to offer clinical manifestation and management of sigmoid sinus thrombosis and facial palsy secondary to mastoiditis.

Methods: A 72-year-old patient known with right antromastoidectomy in other ENT Clinic, was reffered to our ENT Department with right-sided otalgia, headache and with right facial paralisys (loss of forehead wrinkles and inability to frown, inability to close the right eye, the corner of the mouth pulls down). Computed tomography with contrast administration indicated parafluide accumulations at right mastoid cells and thrombophlebitis modifications in sigmoid right sinus.

Results: This case demonstrates rare but serious sequel of mastoiditis: sigmoid sinus thrombosis and right facial paralysis. Middle ear secretation culture was positive with growth of Pseudomonas aeruginosa. In this case, a right mastoidectomy was associated with large spectrum antibiotics prolonged for 3 months. The anticoagulant therapy was established also.

Conclusions: The particularity of this case lies in that neurological symptoms had a slow recovery and also the difficulty 